\title{
Destruction of peritrophic membrane and its effect on biological characteristics and activity of digestive enzymes in larvae of the Indian meal moth, Plodia interpunctella (Lepidoptera: Pyralidae)
}

\author{
SEYEDE MINOO SAJJADIAN and VAHID HOSSEININAVEH \\ Department of Plant Protection, College of Agriculture and Natural Resources, University of Tehran, Karaj, Iran; \\ e-mails: minoo.sajjadian@ut.ac.ir; vnaveh@ut.ac.ir
}

Key words. Lepidoptera, Pyralidae, Plodia interpunctella, peritrophic membrane, calcofluor, enzyme activity, chitin

\begin{abstract}
The mid gut in insects is usually lined with a peritrophic membrane (PM), which protects it from microbial infections and physical contact with ingested food. Calcofluor is an optical brightener that has strong chitin-binding properties, which disrupt the chitin-protein structure of PM. In this study, we investigated the effects of calcofluor on the structure of the PM, activity of some common digestive enzymes and biological characteristics of the Indian meal moth, Plodia interpunctella (Lepidoptera: Pyralidae). Twenty hours after feeding on a calcofluor-containing diet, the PM was completely eliminated. We recorded significant effects of calcofluor on the activity of digestive enzymes in the mid and hindguts of larvae. In both these regions of the gut, in particular, there were significant increases in the activity of $\alpha$-amylase, $\alpha$-galactosidase and trypsin. The activities of $\alpha$-glucosidase and $\beta$-glucosidase in the midgut of treated larvae were lower than in the control, but were significantly higher in the hindgut of treated larvae. The larvae that fed on on calcofluor for $20 \mathrm{~h}$, took longer to complete their larval and pupal stages, were lighter in weight and fewer reached maturity and laid fewer eggs. Results of this and previous studies highlight the crucial role of PM in enzyme recycling and digestion. PM disruption may negatively affect insect development on the one hand, by decreasing the efficiency of food digestion and, on the other, by increasing the costs associated with an increased enzyme demand and disrupted enzyme recycling mechanism.
\end{abstract}

\section{INTRODUCTION}

The alimentary canal of insects is divided into three distinct regions, the fore, mid and hindgut, based on their embryonic origins, structures and physiological functions. The fore and hindgut are derived from embryonic ectoderm and have an inner lining of a protective layer of cuticle, while the midgut is derived from embryonic mesoderm and lacks the inner cuticular covering. The midgut is, therefore, the main region of the alimentary canal where the primary functions of food digestion and nutrient uptake occur. In direct contact with the gut contents the midgut faces constant physical and chemical challenges from indigested food (Lemaitre \& Miguel-Aliaga, 2013). The insect midgut is usually lined with an acellular semipermeable layer, the peritrophic membrane or matrix (PM) (Zhong et al., 2012). The PM is composed of chitin fibrils held together by chitin-binding proteins, especially peritrophins (Klowden, 2007; Lemaitre and Miguel-Aliaga, 2013). PM surrounds the food eaten by insects and prevents it from coming into direct physical contact with the microvillar cells (Wang \& Granados, 2001). This anatomical structure has an important role in enhancing digestive efficiency (Bolognesi et al., 2008). The PM contains pores, which are permeable to some digestive enzymes and the products of digestion. This selective permeability creates a compartmentalization into an endoperitrophic space and ectoperitrophic space (Klowden, 2007). A relatively wide variation exists in the size of pores among different insects: ranging, for example, from 7-7.5 nm in the moth Erinnys ello (Lepidoptera:
Sphingidae) to 150 and $200 \mathrm{~nm}$ in cockroaches and grasshoppers, respectively (Santos \& Terra, 1986).

During the last decades, the widespread use of chemical pesticides has resulted in many insects becoming resistant and harmful effects on non-target organisms, humans and the environment. Additionally, the direct use of chemical insecticides is not practical against some pests, such as those damaging stored products. These problems have led researchers to explore alternative ways to control pests. The midgut is the primary point of entry of many pathogens, such as entomopathogenic viruses. At the same time, it is an important target for many plant-derived or synthetic metabolites, which can potentially interfere with the normal function of the digestive tract, either by inhibiting the normal activity of digestive enzymes or by interrupting PM formation and structure (Vandenborre et al., 2011). Many studies targeting PM in different insect species have shown that application of binding molecules to chitin may provide a new way to control insects. For example, lectins and Calcofluor enhance virus infections, and increase insect mortality by disrupting the chitin-protein networks and increasing the permeability of PM (Cohen, 1987; Harper et al., 1998; Wang \& Granados, 2000; Mohan et al., 2006).

Calcofluor is a chemical stain with chitin binding properties, which inhibits the formation of PM by disrupting chitin-protein interactions (Klowden, 2007). This substance is a fluorescent probe, which make hydrogen bonds with polysaccharides and is commonly used in clinical studies, such as the rapid diagnosis of acute eosinophilic pneumonia (Hogan et al., 1997; Albani, 2001). The PM proteins 
of the moth, Trichoplusia ni (Lepidoptera: Noctuidae) dissociate when the PM is incubated with $1 \%$ calcofluor in vitro conditions. A similar disruption of PM was recorded in larvae of Spodoptera frugiperda (Lepidoptera: Noctuidae) fed on an artificial diet containing $1 \%$ calcofluor (Bolognesi et al., 2001). Inhibition of PM formation by calcofluor disrupts the midgut defense system and enhance larval susceptibility to baculovirus infection in a number of Lepidoptera, including T. ni, Lymanthria dispar, Helicoverpa zea, Pseudaletia unipuncta and Hyphantria cunea (Wang \& Granados, 2000). Similar patterns of increased susceptibility to entomopathogenic viruses are recorded in other lepidopteran pests, such as Agrotis ipsilon (Boughton et al., 2001), S. frugiperda (Martinez et al., 2003) and $L$. dispar (Dougherty et al., 2006).

The aim of this study was to explore the effects of calcofluor on PM development in the Indian meal moth, Plodia interpunctella (Lepidoptera: Pyralidae), an important polyphagous pest of many stored products worldwide. After feeding on a diet containing calcofluor, the larvae were dissected and the activities of some digestive enzymes ( $\alpha$-amylase, $\alpha$-glucosidase, $\beta$-glucosidase, $\alpha$-galactosidase, $\beta$-galactosidase and trypsin) were evaluated in different parts of the alimentary canal.

\section{MATERIAL AND METHODS}

\section{Insects and isolation of peritrophic membrane}

A stock colony of $P$. interpunctella, which originated from infected pistachios, was established in a laboratory under controlled conditions of $27 \pm 1^{\circ} \mathrm{C}, 60 \pm 5 \%$ R.H. and 12L: 12D. The insects were reared on the artificial diet used by Sait et al. (1997). The diet consisted of $800 \mathrm{~g}$ wheat bran, $160 \mathrm{~g}$ dried brewer's yeast, $200 \mathrm{ml}$ honey and $200 \mathrm{ml}$ glycerol. Calcofluor was obtained from SIGMA-ALDRICH®, CAS Number 4404-43-7.

\section{Effects of calcofluor on PM}

To study the effects of calcofluor on $P$. interpunctella PM, 20 one-day old fourth instar larvae fed on an artificial diet containing $1 \%$ calcofluor $(\mathrm{w} / \mathrm{w})$ were used. During $30 \mathrm{~h}$ of feeding on calcofluor, three larvae were dissected every $5 \mathrm{~h}$ to record the changes in PM structure. In the control, the larvae were fed on an artificial diet lacking calcofluor. The PMs of the larvae were obtained by dissecting midguts of the larvae under a stereoscopic microscope in cold water and the epithelial layer was isolated gently using fine forceps to expose the PMs surrounding the food contents.

\section{Enzyme assays}

Physiological assays were conducted to evaluate the effects of feeding on calcofluor on the activity of general digestive enzymes in different regions of the alimentary canal of $P$. interpunctella larvae. Twenty one-day old fourth instar larvae were fed an artificial diet containing $1 \%$ calcofluor $(\mathrm{w} / \mathrm{w})$. The same numbers of larvae were fed a standard diet without calcofluor, as control. After $24 \mathrm{~h}$ of feeding, the larvae were dissected and the three major regions of gut (i.e. foregut, midgut, and hindgut) were separated in different Eppendorf microtubes. The midgut, itself, was transported onto a frozen Steel plate and divided into three regions (anterior midgut, middle midgut and posterior midgut) using a sterile blade. Our preliminary assays revealed no enzyme activity in the foregut, so we did not include it in this experiment.

The protein concentration of all samples was determined according to the method of Lowry et al. (1951) using bovine se- rum albumin as standard. The activities of some general digestive enzymes, including $\alpha$-amylase, $\alpha$-glucosidase, $\beta$-glucosidase, $\alpha$-galactosidase, $\beta$-galactosidase and trypsin were then quantified in the hindgut and each of the three separated parts of the midgut. Alpha-amylase activity was assayed according to Bernfeld (1955) method using dinitrosalisylic acid (DNS) and 1\% soluble starch as the substrate with slight modifications. Briefly, $10 \mu 1$ enzyme extract was added to $85 \mu 140 \mathrm{mM}$ citrate-phosphate-borate buffer ( $\mathrm{pH}$ 9) and $10 \mu \mathrm{l}$ soluble starch. The process was stopped by adding $50 \mu \mathrm{l}$ of DNS (44 $\mathrm{mM})$ and heating in boiling water for $10 \mathrm{~min}$. The absorbance of the reaction mixture was read at $540 \mathrm{~nm}$ using a microplate reader (ELX 808). In the blanks, the enzyme was added to the reaction mixture after DNS.

The activity of $\alpha$-glucosidase and $\beta$-glucosidase was quantified according to Ramzi \& Hosseininaveh (2010) and Tabatabaei et al. (2011) using $p$-nitrophenyl- $\alpha$-D-glucopyranoside ( $p \mathrm{NaG}, 5 \mathrm{mM}$ ) and $p$-nitrophenyl- $\beta$-D-glucopyranoside $(p \mathrm{NbG}, 5 \mathrm{mM})$ as substrates, respectively. This assay was carried out by incubating 10 $\mu 1$ of the enzyme extract with $85 \mu \mathrm{l}$ of $40 \mathrm{mM}$ citrate-phosphateborate buffer ( $\mathrm{pH} 6$ ) and $5 \mu$ substrates for $30 \mathrm{~min}$. The reaction was stopped by the addition of $50 \mu \mathrm{L}$ of $\mathrm{NaOH}(1 \mathrm{M})$. Optical density was measured at $405 \mathrm{~nm}$ using a microplate reader (ELX 808). In the controls, the enzyme was added to the reaction mixture after the addition of $\mathrm{NaOH}$. The same procedure was used to quantify the activity of $\alpha$-galactosidase and $\beta$-galactosidase using $p$-nitrophenyl- $\alpha$-D-galactoside $(p \mathrm{NP} \alpha \mathrm{Gal}, 5 \mathrm{mM})$ and $p$-nitrophenyl- $\beta$-D-galactoside ( $p \mathrm{NP} \beta \mathrm{Gal}, 5 \mathrm{mM})$ as substrates, respectively.

Trypsin activity was determined using BApNA $\left(\mathrm{N}_{\alpha}\right.$-benzoylL-arginine- $p$-nitroanilide, $20 \mathrm{mM}$ ) as a substrate. Briefly, $5 \mu \mathrm{l}$ of substrate was added to $10 \mu 1$ enzyme extract and $85 \mu 140 \mathrm{mM}$ citrate-phosphate-borate buffer at $\mathrm{pH} 10$. The absorbance was then measured continuously for $30 \mathrm{~min}$ at 3-min intervals at $405 \mathrm{~nm}$.

\section{Effect of PM disruption on biological characteristics}

To study the effects of feeding on calcofluor on biological characteristics of $P$. interpunctella, 20 one-day old fourth instar larvae were placed in a plastic box and reared on artificial diet containing calcofluor $1 \%(\mathrm{w} / \mathrm{w})$. In the control, the larvae were fed on the standard diet without calcofluor. The insects were reared under controlled conditions until adult emergence. During this period, some biological characteristics of the treated larvae were recorded, including larval mortality, pupal mortality, occurrence of deformity in adult moths, the weight of the fully developed fourth instar larvae, pupal weight, adult weight, time of appearance of the first pupae and of adult emergence, oviposition rate and hatching rate were recorded and compared with those recorded for the control.

\section{Data analysis}

Data were analyzed using STATGRAPHICS computer software, Version 5.1. All experiments were carried out with three replicates. Independent $t$-tests were used to compare the average values of enzyme activities and biological characteristics of control and treated larvae.

\section{RESULTS}

\section{Calcofluor effects on PM}

The larvae of $P$. interpunctella fed on calcofluor for $5 \mathrm{~h}$ showed slight changes in PM structure (Fig. 1b). However, after feeding for 10 and $15 \mathrm{~h}$, the formation and structure of PM were obviously disrupted (Fig. 1c and d). After $20 \mathrm{~h}$ of feeding on calcofluor no PM was observed in the treated larvae and the contents of the lumen dispersed throughout 


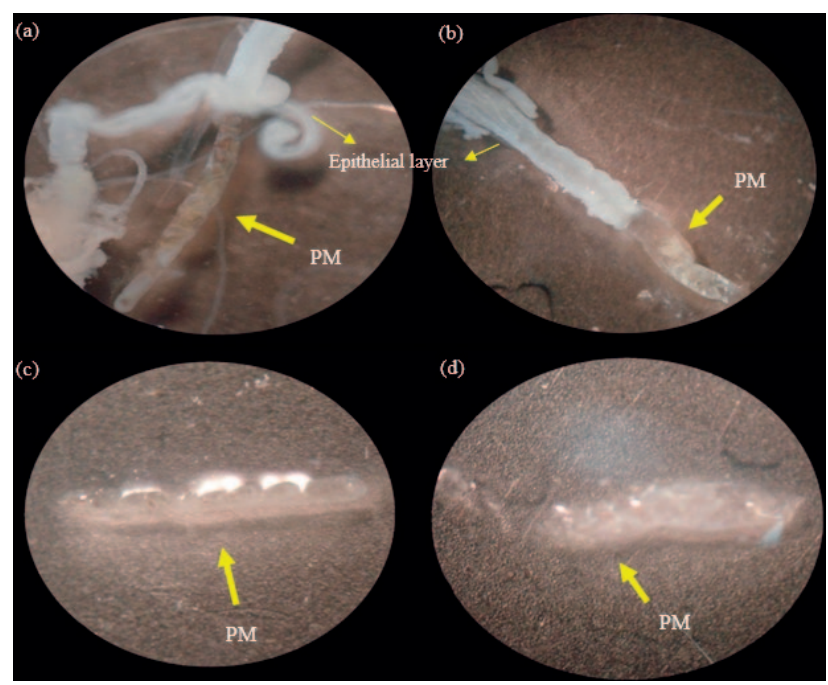

Fig. 1. Peritrophic membrane of Plodia interpunctella larvae: (a) control larvae, (b) 5, (c) 10 and (d) $15 \mathrm{~h}$ after feeding on calcofluor.

the Petri dish when the epithelial layer was removed. In contrast to treated larvae, a normal PM was present in control larvae (Fig. 1a).

\section{Enzyme assays}

The activities of different digestive enzymes in the four separated parts of the alimentary canal of $P$. interpunctella are illustrated in Fig. 2. There was a significant increase in the activity of $\alpha$-amylase in larvae fed on calcofluor in both the hindgut and each of the three midgut sections (Fig. $2 a)$. The activities of $\alpha$-glucosidase and $\beta$-glucosidase were suppressed in the different parts of the midgut of larvae fed on calcofluor. However, a significant increase in activity of these two enzymes was recorded in the hindgut of treated larvae compared to the control (Fig. 2b and c). Disruption of PM by calcofluor caused a significant increase in $\alpha$-galactosidase activity in the hindgut and each of the three midgut sections (Fig. 2d). Although, an increase in activity of $\beta$-galactosidase was recorded in all sections of the alimentary canal of treated larvae studied, this increase was only statistically significant for the anterior and middle parts of the midgut (Fig. 2e). Finally, a significant increase in activity of trypsin was recorded in all four sections of alimentary canal of $P$. interpunctella treated with calcofluor (Fig. 2f).

\section{Effect of PM disruption on biological characteristics}

The fourth instar larvae of the same age (one-day old) were used to study the effects of calcofluor on some biological characteristics of $P$. interpunctella. We recorded a significant increase in development time of both larvae and pupae of larvae fed on calcofluor for $24 \mathrm{~h}$ (Table 1). The control larvae developed into adults within 5 days, while treated larvae took an additional 8 days. The weights of fully developed fifth instar larvae, pupa and adult moths were also affected by calcofluor, with the larvae fed on calcofluor weighing less, as larvae, pupae and adults (Table 1). While we recorded no larval and pupal mortality or adult deformity in the control, an average larval and pupal mor-
TABLE 1. Effects of calcofluor on biological characteristics of $P$. interpunctella. ${ }^{\text {ns }}$ - non-significant; $*$ and ${ }^{* *}$ - significant at 0.05 and 0.01 levels, respectively.

\begin{tabular}{lcc}
\hline Biological characteristic & Control & Calcofluor \\
\hline Larval mortality & 0 & $88.8 \% * *$ \\
Pupal mortality & 0 & $44.4 \% * *$ \\
Adult deformity & 0 & $44.4 \% * *$ \\
Last larval instar weight (mg) & $143 \pm 0.33$ & $122 \pm 0.33^{* *}$ \\
Pupal weight (mg) & $9 \pm 0.33$ & $7 \pm 0.33^{*}$ \\
Adult weight (mg) & $10 \pm 0.33$ & $7 \pm 0.33^{* *}$ \\
Appearance of first pupae (day) & 5 & 8 \\
Appearance of first adult (day) & 3 & 5 \\
Oviposition rate (egg/female) & $91 \pm 5$ & $46 \pm 3^{* *}$ \\
Hatching rate (\%) & $76 \pm 2.3$ & $73 \pm 2.02^{\text {ns }}$ \\
\hline
\end{tabular}

tality of $88.8 \%$ and $44.4 \%$ was recorded for the larvae fed on calcofluor. In addition, on average $44.4 \%$ of the treated larvae showed some form of body deformity (Table 1).

The number of eggs laid by $P$. interpunctella was also affected by calcofluor (t-test: $\mathrm{df}=4, \mathrm{t}=7.22, \mathrm{P}<0.01$ ). During the first three days after adult emergence, on average 91 and 46 eggs per female were laid by the adult moths in the control and calcofluor treatments, respectively. The percentage of eggs that hatched, however, was not affected by calcofluor ( $\mathrm{t}$-test: $\mathrm{df}=4, \mathrm{t}=0.85, \mathrm{P}=0.44$ ).

\section{DISCUSSION}

Although the harmful effects of synthetic pesticides on humans, the environment and non-target organisms such as natural enemies and the resurgence of pests that become resistant to pesticides has been recognized for more than 60 years (Rodriguez et al., 2003; Regnault-Roger et al., 2004), chemical pesticides continue to be the main method of controlling pests (Casey Sclar, 1994). Recent advances in biotechnology and molecular biology have provided new insights into the use of alternative control strategies in line with the integrated pest management (IPM) concept. The genetically engineered crop plants expressing insecticidal toxins, for example, have led to significant reduction in pesticide usage and lower production costs worldwide (Vandenborre et al., 2011).

The chitin-protein structure of the PM protects insects against food particles as well as invasive microorganisms such as entomopathogenic viruses. The chitin fibrils are held together by a variety of chitin-binding proteins (Klowden, 2007; Lemaitre \& Miguel-Aliaga, 2013). Other organic or synthetic compounds with a chitin-binding tendency may compete with normal proteins for these biding sites and so dissociate the normal chitin-protein structure of PM and change the physiology of the alimentary canal in insects.

In this study, the effects of the optical brightener, calcofluor, on the structure of the PM, activity of digestive enzymes and some biological characteristics of the Indian meal moth, $P$. interpunctella, an important polyphagous pest of stored products worldwide, were investigated. Physiological studies show that certain brighteners, such as calcofluor, can strongly bind $\beta$-glucans such as chitin. 

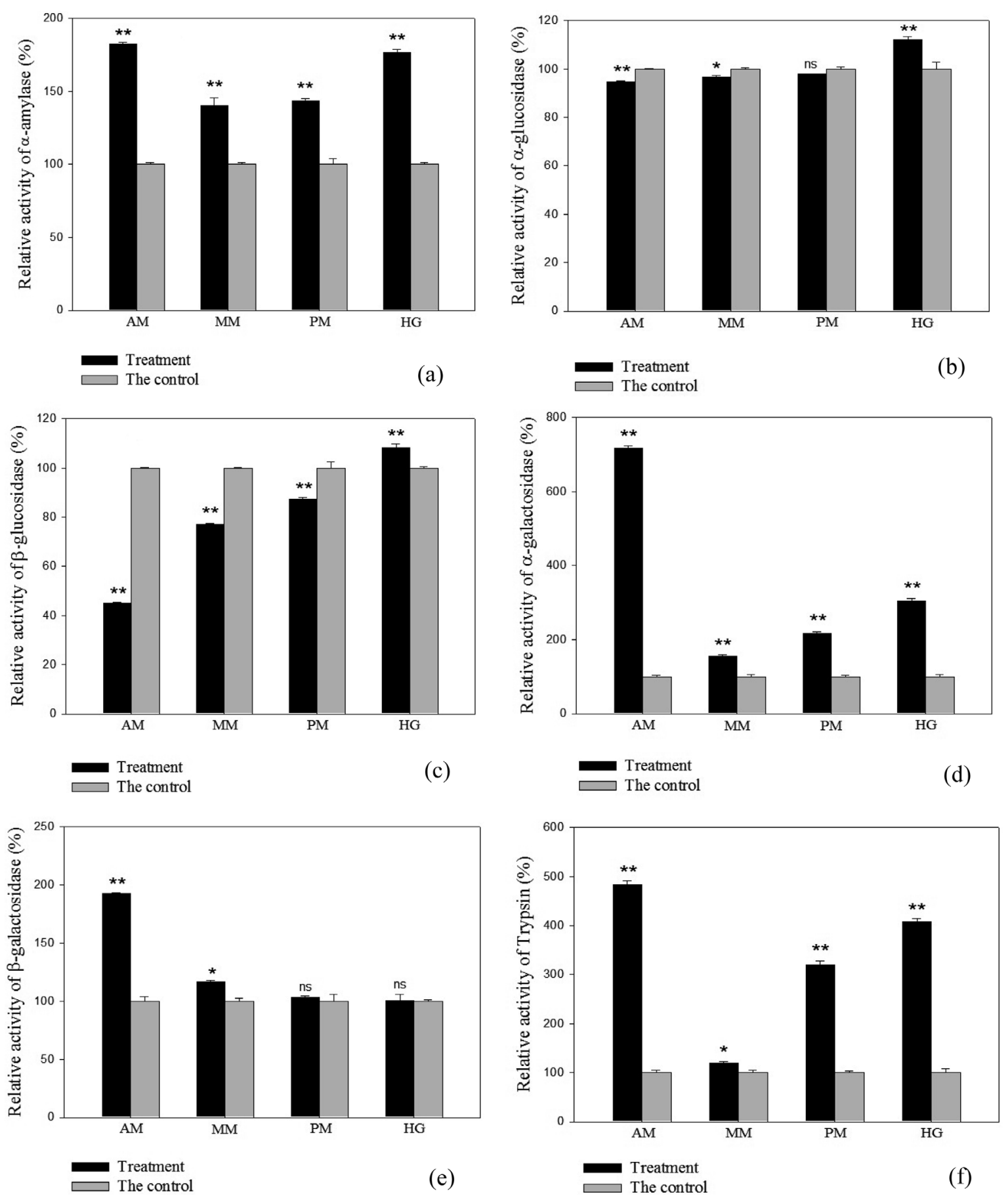

Fig. 2. Activity of $\alpha$-amylase (a), $\alpha$-glucosidase (b), $\beta$-glucosidase (c), $\alpha$-galactosidase (d), $\beta$-galactosidase (e) and trypsin (f) in the mid and hindguts of $P$. interpunctella larvae fed on calcofluor. AM - anterior midgut; $\mathrm{MM}$ - middle midgut; PM - posterior midgut; $\mathrm{HG}-$ hindgut, ${ }^{* *}-\mathrm{p}<0.01 ; *-\mathrm{p}<0.05 ; \mathrm{ns}-\mathrm{p}>0.05$.

Therefore, optical brighteners are likely to increase susceptibility to pathogenic viruses by disrupting the chitin-protein structure of PM of insects (Wang \& Granados, 2000).

Visible effects of calcofluor on the structure of the PM of $P$. interpunctella larvae were recorded over time. Twenty hours after feeding on calcofluor-containing diet, the PM was completely destroyed and the food content distributed throughout the gut without any protective membrane. Disruption of the structure of the PM by calcofluor is previously recorded in some other species of Lepidoptera, such as S. frugiperda (Bolognesi et al., 2001), S. exigua (Zhu et al., 2007) L. dispar, H. zea, T. ni, P. unipuncta and $H$. cunea (Wang \& Granados, 2000). Bolognesi et al. (2001), for example, report that $20 \mathrm{~h}$ of exposure of $S$. frugiperda to calcofluor is enough to completely disrupt the PM.

Calcofluor had significant effects on the activity of digestive enzymes in the mid and hindguts of $P$. interpunctella larvae. Similarly, Sobotnik et al. (2008) show that destruction of PM using calcofluor can result in the suppression of population growth in Acarus siro, an important pest of stored products. Particularly, the activity of $\alpha$-amylase, $\alpha$-galactosidase and trypsin in both the mid and 
hindguts of treated larvae increased significantly. The activities of $\alpha$-glucosidase and $\beta$-glucosidase recorded in the midgut of treated larvae were lower than in the control, but the activity of these two enzymes in the hindgut of treated larvae was significantly increased (Fig. 2). The increased activity of nearly all of the enzymes (with the exception of $\beta$-galactosidase) in the hindgut of treated larvae may be explained by the fact that these enzymes pass from the midgut into the hindgut in absence of a PM, probably as a consequence of the disruption in the compartmentalization of midgut space. These findings highlight the crucial role of the PM in enzyme recycling, i.e. prevention of digestive enzymes being transferred into the hindgut. The increased transfer of digestive enzymes such as amylase, chymotrypsin and trypsin from the midgut to hindgut occurs in $S$. frugiperda larvae whose PM is destroyed by calcofluor (Bolognesi et al., 2008). Similarly, Bolognesi et al. (2001) show that $S$. frugiperda larvae fed on a diet containing $1 \%$ calcofluor, experience a significant increase in trypsin and chymotrypsin enzymes in the middle and posterior portions of the midgut compared to control larvae, which routinely show a decreasing gradient in these enzymes along the midgut. However, they did not evaluate the concentrations of these enzymes in the hindgut.

Our results revealed that calcofluor had significant effects on the development times of both larvae and pupae, weights of the different stages, mortality of larvae and pupae, and oviposition of adults (Table 1). Wang \& Granados (2000) also show that continuous feeding on calcofluor results in a prolonged development time and increase in mortality in $T$. $n i$ larvae. In addition, the pupae and adult moths that emerge from treated larvae are smaller than the controls (Wang \& Granados, 2000). The impaired growth and development of lepidopterous larvae treated with optical brighteners probably results from an increase in the costs associated with the conversion of food into body mass (Bolognesi et al., 2008). The PM has an important role in enzyme recycling and digestion in a compartmentalized midgut. PM disruption may negatively affect insect development, on one hand, by decreasing the efficiency of food digestion and, on the other hand, by increasing the costs associated with increased enzyme demand and the disruption of the enzyme recycling mechanism.

Optical brighteners, such as calcofluor, have frequently been shown to increase the pathogenicity of entomopathogenic viruses by either increasing protection against UV radiation and/or making the midgut susceptible to invasion. These compounds are currently used as synergists to increase the efficiency of biological control by entomopathogenic viruses. Results of the current study, however, together with those of previous studies (e.g. Bolognesi et al., 2001, 2008), clearly indicate that calcofluor, by itself may negatively affect the normal physiology and development of insects, simply by disrupting the PM, which is an important center for food digestion. For example, although the larvae of $T$. $n i$ treated with calcofluor, have an increased susceptibility to artificial viral infection, examination of those larvae, which died after feeding on calcofluor, re- vealed no signs of microbial infection (Bolognesi et al., 2008). These findings provide new insights into the use of these compounds in stored product pest management, either singly or in combination with other control agents.

\section{REFERENCES}

Albani J.R. 2001: Effect of binding of Calcofluor White on the carbohydrate residues of $\alpha_{1}$-acid glycoprotein (orosomucoid) on the structure and dynamics of the protein moiety. A fluorescence study. - Carbohydr. Res. 334: 141-151.

Bernfeld P. 1955: Amylases, $\alpha$ and $\beta$. - Meth. Enzymol. 1: 149-158.

Bolognesi R., Ribeiro A.F., Terra W.R. \& Ferreira C. 2001: The peritrophic membrane of Spodoptera frugiperda: secretion of peritrophins and role in immobilization and recycling digestive enzymes. - Arch. Insect Biochem. Physiol. 47: 62-75.

Bolognesi R., Terra W.R. \& Ferriera C. 2008: Peritrophic membrane role in enhancing digestive efficiency theorical and experimental models. - J. Insect Physiol. 54: 1413-1422.

Boughton A.J., Lewis L.C. \& Bonning B.C. 2001: Potential of Agrotis ipsilon nucleopolyhedrovirus for the suppression of the black cutworm (Lepidoptera: Noctuidae) and effect of an optical brightener on virus efficacy. $-J$. Econ. Entomol. 94: 1045-1052.

Casey Sclar D. 1994: Neem: Mode of Action of Compounds Present in Extracts and Formulations of Azadirachta indica Seeds and their Efficacy to Pests of Ornamental Plants and to Non-target Species. Retrieved October 2005 from: http://www. colostate.edu/DeptsEntomology/Courses/en570/Papers_1994/ sclar.html

COHEN E. 1987: Chitin biochemistry: synthesis and inhibition. Annu. Rev. Entomol. 32: 71-93.

Dougherty E.M., Narang N., Loeb M., Lynn D.E. \& Shapiro M. 2006: Fluorescent brightener inhibits apoptosis in baculovirusinfected gypsy moth larval midgut cells in vitro. - Biocontr. Sci. Technol. 16: 157-168.

Harper M.S., Hopkins T.L. \& Czapla T.H. 1998: Effect of wheat germ agglutinin on formation and structure of the peritrophic membrane in European corn borer (Ostrinia nubilalis) larvae. — Tissue Cell 30: 166-176.

Hogan T.F., Riley R.S. \& Thomas J.G. 1997: Rapid diagnosis of acute eosinophilic pneumonia (AEP) in a patient with respiratory failure using bronchoalveolar lavage (BAL) with calcofluor white (CW) staining. - J. Clin. Lab. Anal. 11: 202-207.

KLowden M.J. 2007: Physiological Systems in Insects. Academic Press, Elsevier, London, 699 pp.

Lemaitre B. \& Miguel-Aliaga I. 2013: The digestive tract of Drosophila melanogaster. - Annu. Rev. Genet. 47: 377-404.

Lowry O.H., Rosembrough N.J., Farr A.L. \& Randdall R.J. 1951: Protein measurement with the Folin phenol reagent. J. Biol. Chem. 193: 267-275.

Martinez A.M., Simon O., Williams T. \& Caballero P. 2003: Effect of optical brightners on the insecticidal activity of a nucleopolyhedrovirus in three instars of Spodoptera frugiperda. —Entomol. Exp. Appl. 109: 139-146.

Mohan S., Ma P.W.K., Pechan T., Bassford E.R., Williams W.P., \& LutHe D.S. 2006: Degradation of the S. frugiperda peritrophic matrix by an inducible maize cysteine protease. $-J$. Insect Physiol. 52: 21-28.

RAMZI S. \& HosseininaveH V. 2010: Biochemical characterization of digestive $\alpha$-amylase, $\alpha$-glucosidase and $\beta$-glucosidase in pistachio green stink bug, Brachynema germari Kolenati (Hemiptera: Pentatomidae). - J. Asia-Pac. Entomol. 13: 215-219. 
Regnault-Roger C., Staff V., Philogène B., Terrón P. \& VinCENT C. 2004: Biopesticidas de Origen Vegetal. Mundi-Prensa, Madrid, $313 \mathrm{pp}$.

Rodríguez C., Silva G. \& Duair V. 2003: Insecticidas de origen vegetal. In: Bases para el Manejo Racional de Insecticidas. Fundación para la Innovación Agraria, Facultad de Agronomía, Universidad de Concepción, Chile, pp. 89-111.

Sait S.M., Begon M., Thompson D.J., Harvey J.A. \& Hails R.S. 1997: Factors affecting host Selection in an insect host-parasitoid interactions. - Ecol. Entomol. 2: 225-230.

SANTOS C.D. \& TerRa W.R. 1986: Distribution and characterization of oligomeric digestive enzymes from Erinnyis ello larvae and inferences concerning secretory mechanisms and the permeability of the peritrophic membrane. - Insect Biochem. 16: 691-700.

Sobotnik J., Kudlikova-Krizkova I., Nancova M., Munuzbergova Z. \& HUBert J. 2008: Chitin in the peritrophic membrane of Acarus siro (Acari: Acaridae) as a target for novel acaricides. - J. Econ. Entomol. 101: 1028-1033.

Tabatabaei P.R., Hosseininaveh V., Goldansaz S.H. \& Talebi K. 2011: Biochemical characterization of digestive proteases and carbohydrases of the carob moth, Ectomyelois ceratoniae (Zeller) (Lepidoptera: Pyralidae). — J. Asia-Pac. Entomol. 14: 187-194.

Vandenborre G., Smagghe G. \& Van Damme E.J.M. 2011: Plant lectins as defense proteins against phytophagous insects. Phytochemistry 72: 1538-1550.

Wang P. \& Granados R.R. 2000: Calcofluor disrupt the defense system in insects. - Insect Biochem. Mol. Biol. 30: 135-143.

WANG P. \& GRANADOS R.R. 2001: Molecular structure of the peritrophic membrane (PM): Identification of potential PM target sites for insect control. - Arch. Insect Biochem. Physiol. 47: $110-118$.

Zhong X., Zhang L., Zou Y., Yi Q., Zhao P., Xia Q. \& Xiang Z. 2012: Shotgun analysis on the peritrophic membrane of the silkworm Bombyx mori. — Biochem. Mol. Biol. 45: 665-670.

Zhu R., Liu K., Peng J., Yang H. \& Hong H. 2007: Optical brightener M2R destroys the peritrophic membrane of Spodoptera exigua (Lepidoptera: Noctuidae) larvae. - Pest Manag. Sci. 63: 296-300.

Received August 11, 2014; revised and accepted December 22, 2014 Prepublished online February 27, 2015 